\title{
The Development of Geomagnetic and Auroral Storms
}

\author{
Syun-Ichi AKASOFU \\ Geophysical Institute, University of Alaska, College, Alaska
}

\begin{abstract}
The development of the main phase of geomagnetic storms and of magnetic and auroral substorms is examined on the basis of analyses of individual geomagnetic storms. Several important aspects of geomagnetic storms revealed in this study are reported and discussed.
\end{abstract}

\section{Introduction}

In this paper, an attempt is made to study individual magnetic storms and auroras instead of their average properties. This is because a study of the average magnetic storm is only the first step towards a better understanding of geomagnetic storms.

The earlier studies were, however, based on rather limited amounts of material. The IGY/C made much more abundant material available. The concept of geomagnetic storms should thus be advanced beyond the earlier pioneer works by Chapman and others.

This is a report of work based on such a principle. The work has revealed several important aspects of geomagnetic and auroral substorms that should be coordinated with the earlier concept. Such new aspects are of vital importance in constructing theoretical models.

\section{Low Latitude D, Dst and DS Fields}

Chapman (1919, 1927, 1935, 1952) resolved the storm field $D$ into the combination of an axially symmetric part $D s t$ and an asymmetric part $D S$, namely

$$
D=D s t+D S
$$

Here, $D S$ denotes the disturbance local-time inequality and its mean is the solar disturbance day daily variation $S D$.

Chapman's analysis is equivalent to a Fourier analysis of the $D$ field

$$
D=c_{0}+\sum_{n} c_{n} \sin \left(n \lambda+\varepsilon_{n}\right)
$$

where $\lambda$ denotes $d p$ longitude and $\varepsilon_{n}$ the phase angle. The first term indicates the d.c. component and the second term the harmonic a.c. components. By definition, then

$$
\begin{aligned}
& D s t=c_{0} \\
& D S=\sum_{n} c_{n} \sin \left(n \lambda+\varepsilon_{n}\right) \\
& c_{1} \gg c_{2}, c_{3}, \ldots \ldots
\end{aligned}
$$

On this basis, he constructed the well-known equivalent current systems that could produce the observed $D, D s t$ and $D S$; the current systems are assumed to be located on a single spherical shell concentric with and above the earth, although the actual current systems 
are likely to be three dimensional.

However, the procedure of constructing model current systems for polar magnetic substorms and for the ring currents from the above precisely defined equivalent current systems is unfortunately rather arbitrary. The model current system for polar magnetic substorms is assumed to consist of the whole $D S$ current system and a part of the polar Dst current system; and the whole currents are assumed to be located in the ionosphere. The model current system for the ring current is assumed to be located at least a few earth radii away from the earth's center, encircling the earth axially symmetrically, producing the major part of Dst in low and middle latitudes.

This is equivalent to assuming that the axial asymmetry of $D$ in low latitudes is entirely due to the return currents from the polar electrojets. The westward electrojet produces an eastward return current and thus a positive $H$ change in the morning sector; and the eastward jet produces a westward return current and thus a negative $H$ change in the afternoon sector.

However, Akasofu and Chapman (1964) have shown that the asymmetry of $D$ in low latitudes is not necessarily due to the return currents for several major magnetic storms during the IGY/C. Figure 1 shows iso-intensity contours of $D(H)$ at the maximum epoch $(1000 \mathrm{UT})$ of the great magnetic storm of February 10, 1958. The auroral zone was fairly quiet at that time and there was no significant electrojet along the zone. Even if the electrojets (causing both positive and negative bays) of magnitude as large as $500 \gamma$ existed between $60^{\circ}$ and $70^{\circ} d p$ latitude, its effect in low latitudes was not more than

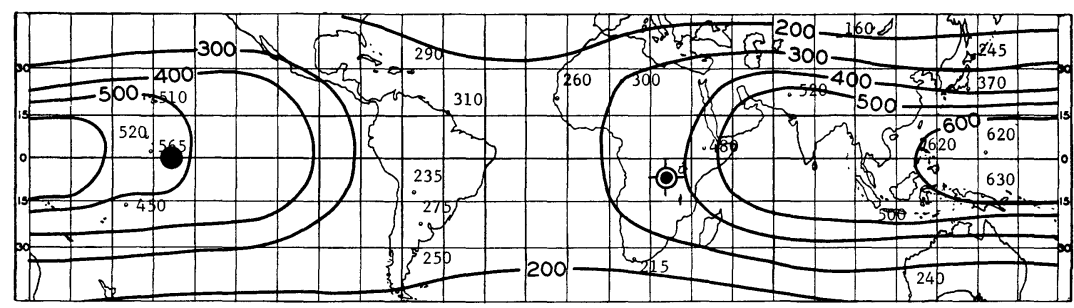

Fig. 1 The distribution of the horizontal component of the disturbance field $D(H)$, at 1000 GMT, 11 February 1958. The subsolar point is indicated by a rayed open circle, the anti-subsolar point by a black circle.

$$
2 c_{1}<2 \times 500 \gamma \times\left(\frac{10^{\circ}}{60^{\circ}}\right) \times \frac{1}{2} \simeq 80 \gamma
$$

where half of the return current is supposed to flow in the middle and low latitude belt $\left(0^{\circ} \sim\right.$ $60^{\circ} \mathrm{dp}$ lat) and the other half over the polar cap. The above value of $80 \mathrm{r}$ is much less than the actual asymmetry of order $300 \gamma$.

Figure 2 also shows iso-intensity contours of the $D(H)$ field at 08 and 09 UT during the great magnetic storm of September 13, 1957, together with the $H$ records from several stations during this period. At 08 UT the auroral zone was fairly quiet, but the low latitude $D$ field showed a considerable asymmetry. Then a very intense westward electrojet began to grow at about $0815 \mathrm{UT}$; it produced negative bays of magnitude more than $1000 \gamma$ at College, Sitka and Cape Wellen, and of a few hundred gammas at Tixie Bay and Dixon; the locations 


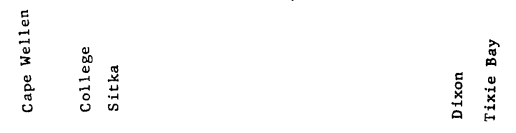

(a)

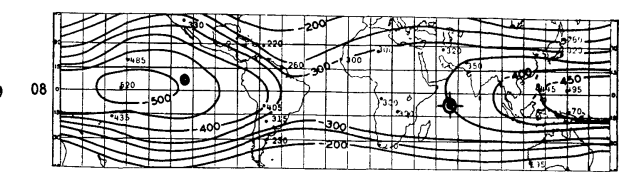

(b)

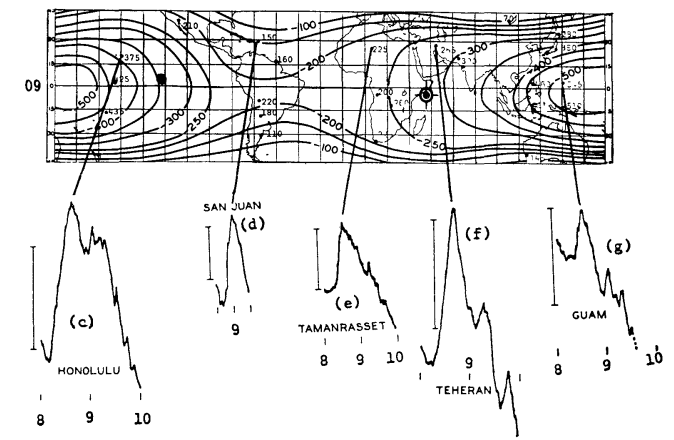

Fig. 2 The distribution of the $D(H)$ field during the great magnetic storm of 13 September 1957. At $0800 \mathrm{UT}$, the auroral zone was fairly quiet; at about $0815 \mathrm{UT}$, an intense substorm began, and it was recorded at the auroral zone stations, indicated at the top of the figure. The positive bays associated with substorm, and recorded at Honolulu, San Juan, Tamanrasset, Teheran and Guam are shown at the bottom of the figure.

of these auroral zone stations are indicated at the top of the figure. The jet subsided quickly from about 09 UT. If the major portion of the asymmetry (namely, the $D S$ ) is produced by the return currents in the way suggested by the early model current system for polar magnetic substorms, the asymmetry or the amplitude of the a.c. component $\left(c_{n}\right)$ should be increased during an enhanced polar magnetic substorm. On the contrary, positive bays were observed in all longitudes, indicating that in this case the major part of the axial asymmetry was not due to the return current, and further the assumption $c_{1} \gg c_{0}$ is not correct in low latitudes. In fact, the basic pattern of the asymmetry grew steadily with the growth of the major storm phase and was affected only a little by intermittent and rapid growth and decay of polar magnetic substorms.

Akasofu, Chapman and Meng (1965) have recently proposed an important revision for the earlier model current system of the polar magnetic substorm. In their new model, the polar electrojet is usually westward in all longitudes, and lies along an oval which is not concentric with the auroral zone (Figure 3). The center of this oval is appreciably displaced from the auroral zone towards the darkside roughly along the midnight meridian. The auroral zone coincides approximately with the average location of this oval only in the midnight sector, where the aurora appears most frequently; because of the daily rotation of the earth, the geographic locus of active aurora, which by definition is the auroral zone, has a 


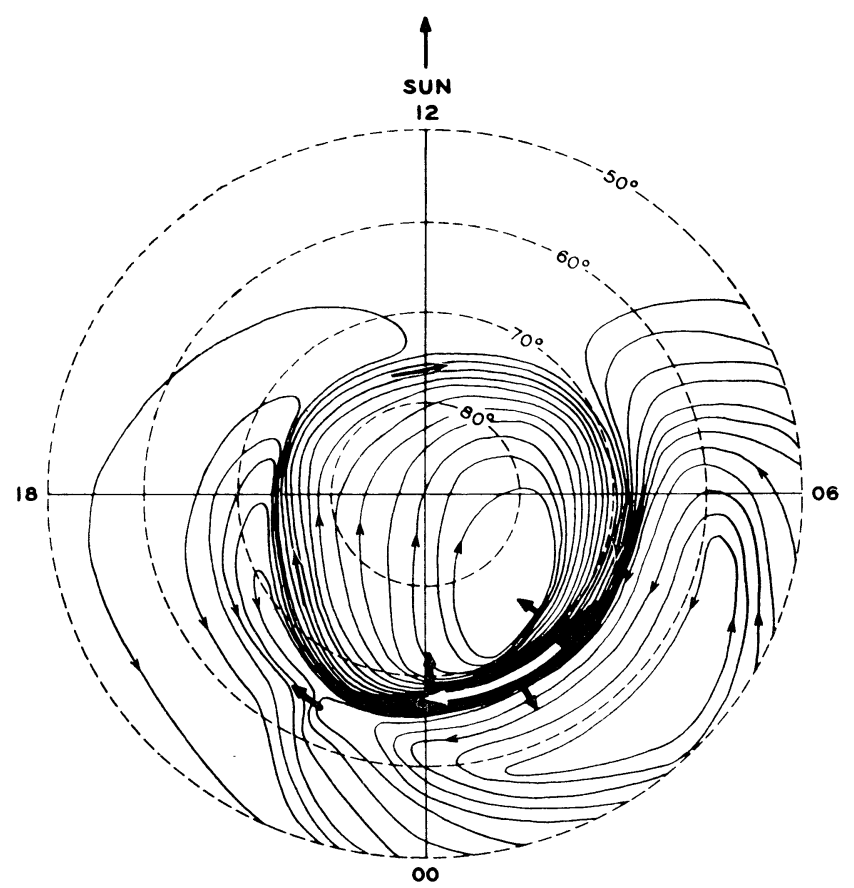

Fig. 3 A new model current system for the polar magnetic substorm; view from above $d p$ north pole; the direction of the sun is indicated.

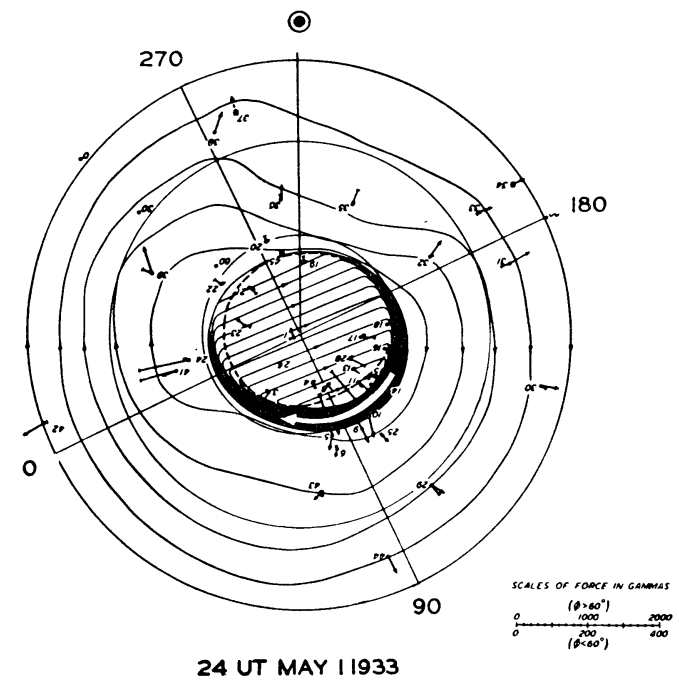

Fig. 4 Mean hourly disturbance vectors and corresponding equivalent current system at 24 UT, 1 May 1933; view from above $d p$ north pole; the direction of the sun is indicated.

more nearly circular pattern. The flow pattern of the polar electrojet coincides approximately with the instantaneous belt of the auroras, rather than along the statistically determined 
auroral zone. A significant current departs from the main westward jet and flows from the afternoon sector across the midnight meridian, rejoining the main jet in the morning sector; such a return current produces positive bays over a large part of the earth's surface. These may be particularly intense in the afternoon sector along the auroral zone and were formerly interpreted as indicating the existence of the supposed eastward electrojet.

The major axial asymmetry of the $D(H)$ field in the interzonal belt is thus not likely to be due to the return currents; the return current may contribute to it when the jet is enhanced, but its contribution cannot be large and further cannot be expressed by the a.c. components alone, and there should be a significant d.c. component $\left(c_{0} \approx c_{n}\right)$. Figure 4 shows one of the equivalent current systems drawn by Vestine (1940) for the magnetic storm of March 31, 1933; the diagram clearly indicates that the electrojet cannot be the major cause for the asymmetry of the $D$ field in the interzonal belt; the low latitude current lines represent the combined $D s t$ and $D S$ variations; their notable deformation in the daytime forenoon sector indicates a positive $D S$ change added to the $D s t$ fields, but this asymmetry cannot be due to the return current from the westward polar jet which at that time was located mainly in the midnight sector. A new current system must be called for the asymmetry. Such a serious problem has become apparent only after a number of individual magnetic storms were extensively examined.

\section{An Indented Ring Current}

Based on the finding that there must be a fairly steady current system which contributes a substantial portion of the asymmetry in the $D$ field, independent of the growth and decay of auroral electrojets, Akasofu and Chapman (1964) have proposed a model current

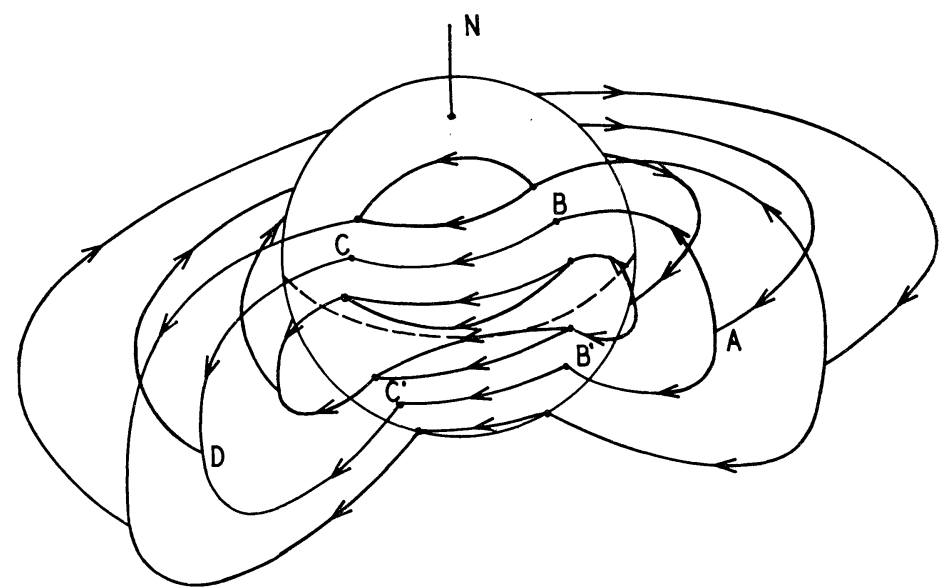

Fig. 5 Schematic drawing to illustrate the current system for the storm main phase. The ring current close its current along an indented path through the ionosphere. The arrow indicates the direction of motions of positive ions in the current system. 
system, partly external and partly ionospheric, which accounts for the asymmetric $D$ field in low and middle latitudes (Figure 5). Their model is also based on the fact that the growth of asymmetry is closely related to that of the Dst component.

Such a current system could be set up if 'ring current particles', both positive and negative, are produced on the dayside, so that the ring current belt will have a gap on the nightside. On the dayside of the ring current belt, there will be electrical neutrality, because positive ions and electrons should exist in equal numbers. However, as soon as the protons start to drift westward and the electrons eastward, charges will tend to accumulate at the gap ends, giving a positive charge on the morning end ( $A$ in Fig. 5) and a negative charge on the evening end $(D)$.

Such charges will be discharged along the field lines of force (from $A$ to $B$ and $B^{\prime}$ ) and in the ionosphere. There the current will flow westward from $B$ to $C$ (and $B^{\prime}$ to $C^{\prime}$ ) on the nightside and eastward on the dayside. (In order to avoid complication, the current lines on the dayside are not drawn in Fig. 5.) At the evening end, the current flows from $C$ and $C^{\prime}$ to $D$. Fig. 5 shows paths of positive ions schematically.

Possible sources of the energy for such an indented ring circuit are neutral hydrogen atoms. If the storm-producing solar plasma contains at times an appreciable amount of hydrogen atoms with energy of order $5 \mathrm{kev}$, these atoms can penetrate directly to the upper part of the ionosphere. There they collide with atmospheric particles $(M)$ and may become energetic protons through the reaction such as

$$
H+M \rightarrow H^{+}+e+M
$$

As soon as the reaction takes place, the motion of the resulting protons is controlled by the geomagnetic field. Some of them will be temporarily 'trapped' and become ring current particles. The reaction occurs primarily on the dayside.

The volume $V$ generated by rotating a line of force which crosses the equatorial plane at $r=r_{e}$ around the dipole axis is given by

$$
V=0.457 \times\left(\frac{4 \pi}{3}\right) r_{e}^{3}
$$

Thus, the volume $d V$ of the shell generated by rotating two lines of force $r_{e}$ and $r_{e}+d r_{e}$ is

$$
d V=5.74 r_{e}^{2} d r_{e}
$$

For simplicity, we assume that the above reaction takes place on a spherical surface, concentric with the earth, of radius $r(=6870 \mathrm{~km})$. Let $p$ be the total number of the protons thus injected with the equatorial pitch-angle $\theta_{e}$ into the shell $r_{e}$ of the thickness $1 \mathrm{~cm}$ at the spherical surface at latitude $\phi$. It is given by

$$
p=2 \times(S \cdot F) \times(2 \pi-\Omega) r \cos \phi
$$

where $F$ denotes the flux of the neutral hydrogen atoms contained in the plasma flow, $S$ the injection coefficient or the efficiency of the injection on the formation of the ring current belt, $\Omega$ the angle in radian of the gap (AOD in Fig. 5), $\phi$ the $d p$ latitude.

The total number $N_{p}$ of the protons with $\theta_{e}$ in the shell $r_{e}$ can be obtained from 


$$
\frac{d N_{p}}{d t}=p-L
$$

where $L$ denotes the loss rate. One of the major loss processes will be the asymmetric charge exchange with helium atoms, because the belt is likely to be formed in the region where helium is the dominant constituent $(r \simeq 1.2 a \sim 4 a)$;

$$
L=\left(Q \bar{N}_{H e} V\right) N_{p}
$$

where $Q$ denotes the reaction cross section, $\bar{N}_{H e}$ the average number density of helium atoms met by protons with $\theta_{e}$ and $r_{e}$. Terrestrial hydrogen atoms may also contribute to the loss process through $H^{+}+H \rightarrow H+H^{+}$, as suggested by Dessler, Hanson and Parker (1961). The total number $N$ of the protons contained in the belt should be

$$
N=\int N_{p} d V
$$

The belt produced by such 'albedo' protons lies mainly between $r_{e}=1.2 a$ and $r_{e}=2.5 a$ (Akasofu 1964), and the continuity of the current is satisfied by the ionospheric currents illustrated in Fig. 5. Akasofu and Chapman (1964) have shown that the proposed indented ring current can produce a large asymmetry of the $D(H)$ field, $-150 \gamma$ on the dayside and $-320 r$ on the nightside, the ratio being $320 / 150=2.1$.

Such a model ring current system can certainly remove a number of difficulties we have encountered in trying to explain the formation of the belt. We have already seen in Fig. 1 and 2 that the magnitude of the Dst component can be as large as $400 \gamma$. If this is due to the ring current, the ring current belt must be located at about a geocentric distance $r_{e_{0}}=2 a$ ( $a=$ the earth radius) or even less. The table below shows the maximum $D s t$ values expected from particular model belts located at several different geocentric distances (Akasofu, Chapman and Venkatesan (1963; correction, 1964)). The maximum Dst values are obtained by examining the maximum number density of the ring current particles for which at some point within the belt the ring current field reaches the dipole field intensity. It is very likely that the belt will become unstable well before such a condition is reached.

\begin{tabular}{c|c|c|c|c}
\hline$r_{e_{0}}$ & $1.5 a$ & $2 a$ & $4 a$ & $6 a$ \\
\hline $\begin{array}{l}\text { Max Dst }(H) \\
\begin{array}{c}\text { Total kinetic } \\
\text { energy (erg) }\end{array}\end{array}$ & $-510 \gamma$ & $-405 \gamma$ & $-150 \gamma$ & $-45 \gamma$ \\
\hline
\end{tabular}

The main phase of great magnetic storms is composed of two parts, most clearly characterized by their decay rate; one decays much more rapidly ( $6 \sim 12 \mathrm{hr})$ than the other $(2 \sim 7$ days). Because of its rapid decay, the combination with the slow decay part gives a rather sharp change of decay rate, which is commonly seen for great storms. This is also a feature of magnetic storms not brought to light clearly by earlier statistical studies. The expected distance of order $r_{e 0}=2 a$ or even less is also supported by a rather short life of the major part of the main phase, of order only $6 \sim 12$ hours.

Further, the distribution of the $D(H)$ field in Figures 1 and 2 is far from that which is 
expected from a ring current located at about $r_{e_{0}}=6 a$ or from a study of the average storm. Such a distant belt produces a distribution expressed by $D_{0}(H) \cos \phi$ (where $D_{0}(H)$ denotes the $D(H)$ value at the $d p$ equator, and $\phi d p$ latitude). The distribution of the $D(H)$ field should be studied in this way for individual magnetic storms of different intensities.

A direct injection of solar particles to this distance requires particle energies of order $1 \mathrm{Bev}$. Satellite observations outside the magnetosphere indicate that the flux of such particles is far less than that expected from the required rate of the accumulation $1.5 \times 10^{23} / 12 \mathrm{hr}$ $=3.5 \times 10^{18} \mathrm{ergs} / \mathrm{sec}$. Dessler, Hanson and Parker (1961) proposed as an alternative that hydromagnetic shock waves, generated at the magnetospheric boundary, are dissipated in the magnetosphere, and increase the temperature of terrestrial thermal protons there. For this mechanism to work efficiently, the amplitude $\Delta B$ of the hydromagnetic waves must be comparable with $B$ itself. This requires that $\Delta B$ must be order $4000 \mathrm{r}$ if the belt is located at $r_{e 0}=2 a$. It is doutful if such violent hydromagnetic waves can occur at such a distance.

Such difficulties arise because the region where the belt is likely to be formed is well protected by the geomagnetic field from the intrusion of the solar plasma; the difficulties will not be removed even if the belt is formed at a greater distance, say $r_{e}=4 a \sim 5 a$. Therefore, a possibility is that the ring current energy is introduced into the magnetosphere in a form which is not affected by the geomagnetic field. As pointed out already neutral hydrogen atoms, if they are contained in the solar plasma, can be a possible source for the ring current belt.

Again, at this point, it may be worthwhile to examine the development of the main phase of individual magnetic storms, rather than that of the average storm. Akasofu and Chapman (1963) have already shown that the development of the main phase shows a great variety and does not seem to be related to the magnitude of the enhanced plasma pressure. Changes in the plasma pressure, sudden, irregular, positive or negative, all of them, do not seem to contribute in any obvious way to the major phase of magnetic storms (Akasofu, 1964). In fact, a considerable geomagnetic storm can develop without an obvious sign of the enhancement of the solar plasma pressure which is usually manifested by the storm sudden commencement; this type of storm is often called a gradually commencing storm (Sg storm). Figure 6 shows such an example; note the magnitude of the main phase decrease of order $200 \gamma$ (for details, see Akasofu, 1965). Unfortunately, only very little attention has been given to this type of storm in earlier works, although their occurrence is fairly common. This is partly because the absence of ssc does not allow us to determine $D s t$ by the earlier method of the analysis. Obviously, the concept of geomagnetic storms should be extended to include the existence of such storms, since they are not by any means exceptional.

It is known that Sg storms are, in general, weaker than ssc storms ( $c f$. Newton and Milson, (1954)), but it is also true that there are a number of Sg storms which have a greater Dst value than the average $D s t$ value of great magnetic storms (90r).

There is yet another type of magnetic storm which does not show any appreciable enhancement of polar magnetic substorms during a steady initial phase, but do show an intense activity after a sudden decrease of the plasma pressure. The sudden decrease is sometimes followed by a rapid growth of the ring current belt. Again, they are not uncommon. Figure 7 


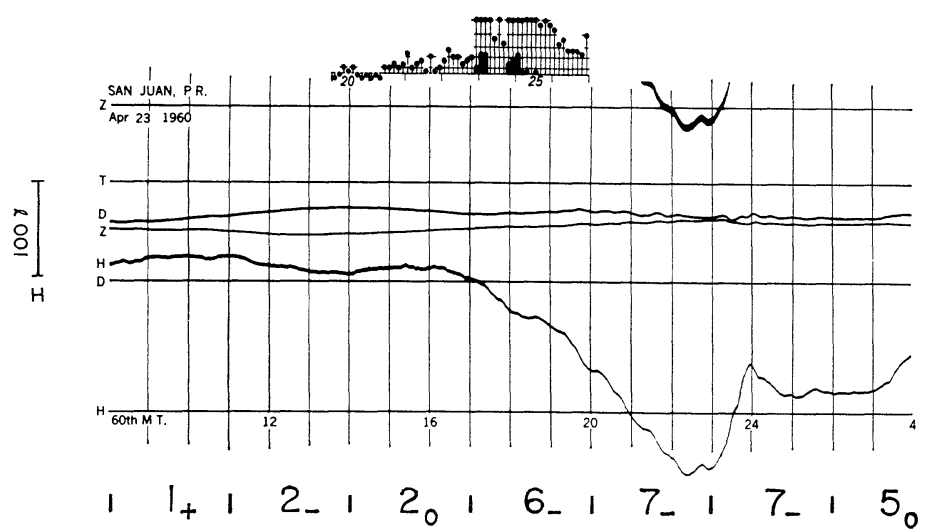

Fig. 6 A typical example of the Sg storm. The musical $K p$ diagram covering the period of the storm is indicated also at the top and bottom.

shows a typical example of such storms. Such a feature cannot be revealed by the average study, since it has been based mostly on hourly mean values of the field components.

Because a positive change in the $H$ component at the time of ssc and during the initial phase is produced by an enhancement of the ionized component of the storm-producing plasma, the fact that there is no obvious relation between the enhancement and the growth of the major storm phase suggests no substantial energy introduction into the magnetosphere to produce the ring current belt by an enhancement of the ionized component of the solar plasma flow.

It may be worthwhile also at this point to refer to the $\Sigma K p$-the average solar plasma velocity $(\bar{v})$ relation obtained by Snyder, Neugebauer and Rao (1963), because our conclusion that changes in the plasma pressure do not contribute in any obvious way to the major phase of magnetic storms seems to apparently contradict with their conclusion.

Certainly, an enhancement of the plasma velocity and thus of the plasma pressure (provided that the plasma density does not decrease greatly) will enhance geomagnetic activity, because the enhanced plasma pressure shrinks the magnetospheric cavity and increases the magnetic field inside the cavity; this field is denoted by DCF (Akasofu and Chapman 1961). However, the $D C F$ field is not the whole storm field; during the major storm phase, the ring current develops, resulting in the $D R$ field and intense polar magnetic substorms grow and decay intermittently (the $D P$ field).

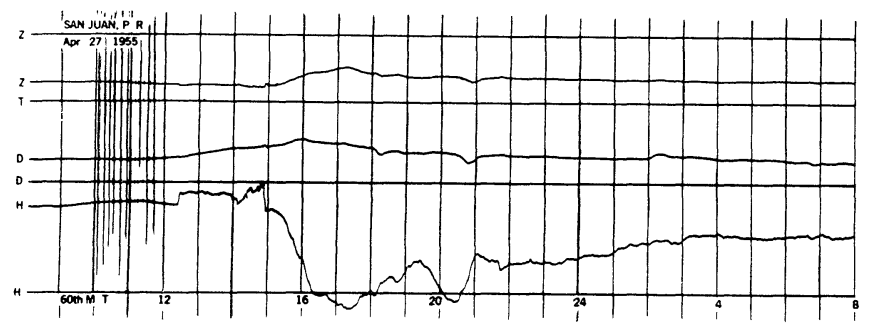

Fig. 7 A typical example of magnetic storm which developed considerably after a sudden decrease in the $H$ component. 
Among them, polar magnetic storms contribute most significantly to the $K p$ index. This can be easily seen from the following list of the observatories contributing to the determination of the $K p$ index (IAGA News No. 3, 1965);

\begin{tabular}{l|c|l|l}
\hline \multicolumn{1}{c|}{ Observatory } & $d p$ lat & Observatory & $d p$ 1at \\
\hline Meanook & $61.8^{\circ} \mathrm{N}$ & Rude Skov & $55.8^{\circ} \mathrm{N}$ \\
Sitka & $60.0^{\circ} \mathrm{N}$ & Wingst & $54.5^{\circ} \mathrm{N}$ \\
Lerwick & $62.5^{\circ} \mathrm{N}$ & Witteveen & $54.2^{\circ} \mathrm{N}$ \\
Eskdalmuir & $58.5^{\circ} \mathrm{N}$ & Hartland & $54.6^{\circ} \mathrm{N}$ \\
Lovö & $58.1^{\circ} \mathrm{N}$ & Agincount & $55.0^{\circ} \mathrm{N}$ \\
Fredericksburg & $49.6^{\circ} \mathrm{N}$ & Amberley & $47.7^{\circ} \mathrm{S}$ \\
\hline
\end{tabular}

They are mostly subauroral belt stations, none of the stations below $d p$ lat. $45^{\circ}$. Obviously, the largest contribution to the $K p$ index is thus due to the $D P$ field; so that large $K p$ values such as 6, 7, 8 and 9 are mainly due to the $D P$ field, rather than the $D C F$ field. Further, a sudden enhancement of the solar plasma pressure does not, in general, cause polar magnetic substorms; Wilson (1964) showed that in both the auroral zone and subauroral belt, complicated oscillatory changes of the field are commonly seen at the time of ssc.

Therefore, the relation $(\Sigma K p-v)$ obtained for rather small values of $K p$ should not be extrapolated to $K p$ values of more than 6 ; the extrapolation suggests the magnitude of ssc as large as $800 \gamma$, corresponding to $K p=9$. Such a large ssc is very unlikely to occur, although $K p=9$ are often experienced during magnetic storms. Further, the $K p$ sum, $\Sigma K p$, is too crude to be used for a detailed study of the development of geomagnetic storms.

Piddington (1964) has proposed that a part of the main phase decrease may be caused by electric currents in the tail region of the magnetosphere. Because the tail region is located beyond $r_{e}=6 a$ (outside the co-rotating region of the magnetosphere), its effect does not seem to be more than $50 \gamma$ at the earth's surface.

Cole (1964a, b) has proposed also an interesting idea that electric polarization fields caused by the polar electrojets in the ionosphere produce an interchange motion of the magnetospheric plasma, resulting in its energization. It would not be difficult to examine for individual storms how many polar magnetic substorms should occur before an appreciable main phase can be recognized.

\section{Solar Plasma Flow in Interplanetary Space}

There are now a few important evidences to suggest that the storm-producing plasma is not simply an expansive motion of the solar corona. This is based on a careful study of the position of solar flares, its associated polar cap absorption and the Forbush decreases for individual events (Haurwitz, Yoshida and Akasofu, 1965). They have found that both a large Forbush decrease and a large increase in PCA at the time of storm sudden commencement are caused almost exclusively by eastern and central flares, but not by western flares. Further, Bell (1961) and Yoshida and Akasofu (1965) have shown a remarkable 'center-limb effect' of the intensity of geomagnetic storms and Forbush decreases; the storms of the 
largest magnitude tend to originate near the central meridian of the solar disk. Haurwitz, Yoshida and Akasofu (1965) proposed that this basic asymmetry in solar terrestrial relationships develops as a natural consequence of the expanding storm-producing plasma and the spiral magnetic field set up by the solar wind (Figure 8); the storm-producing plasma is considered to be ejected from the flare region, the chromosphere, into a rather narrow cone.

If the storm-producing plasma is simply an enhanced expansive motion of the solar corona, namely the blast wave (Parker, 1961), the plasma will contain very little neutral hydrogen atoms. However, it is rather difficult to imagine the blast wave which is confined to propagate in a rather narrow cone to produce not only the remakable center-limb effect, but also the great asymmetry in PCA's and Forbush decreases.

If, on the other hand, the storm-producing plasma originates in the flare region, there is a possibility that the plasma contains an appreciable flux of neutral hydrogen atoms. Akasofu (1964) has already shown that the photo-ionization by the extreme ultraviolet radiation of the sun is not likely to change seriously the initial degree of ionization of the chromospheric plasma during its passage to the earth's distance. If the initial plasma is dense enough, the recombination becomes more important than the photo-ionization and thus the degree of ionization could be reduced, rather than increased. The equation of degree of the ionization $x$ is given ( $c f$. Akasofu, 1964) by

$$
\frac{d x}{d t}=W r(1-x)-\alpha n x^{2}+\delta n(1-x) x
$$

where

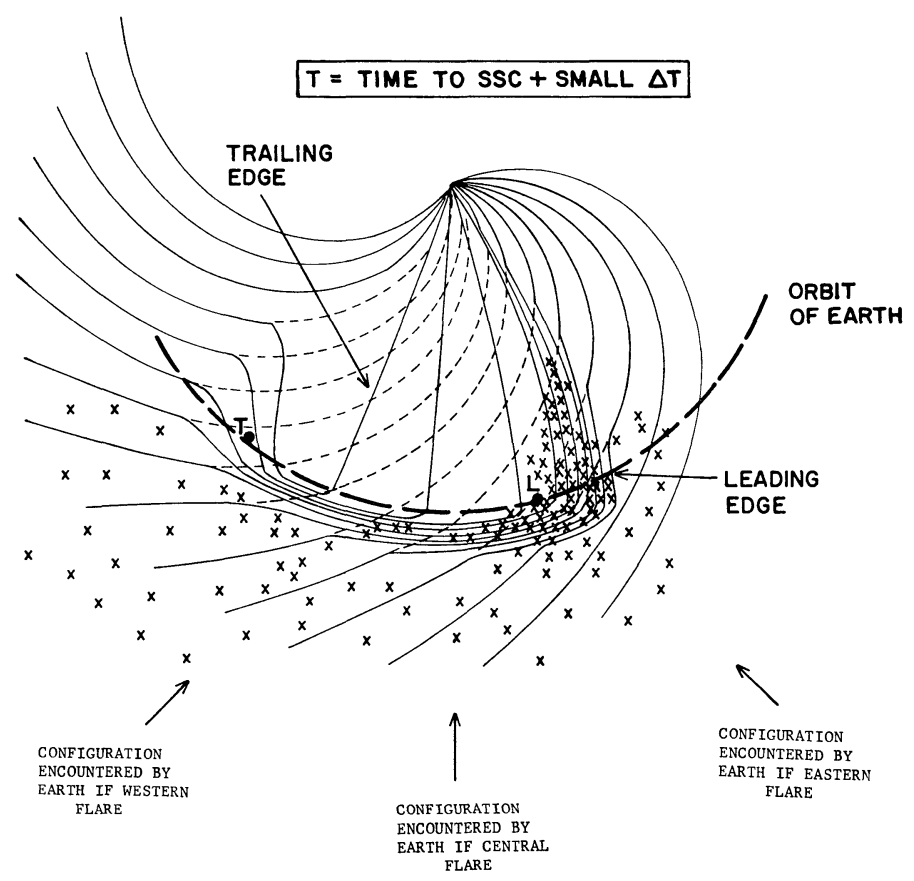

Fig. 8 Schematic diagram to illustrate the asymmetric magnetic field storm producing plasma flow configuration. 


$$
\begin{aligned}
W & =\text { the dilution factor } \\
\gamma & =\text { photo-ionization rate } \\
\alpha & =\text { the recombination rate } \\
\delta & =\text { the collision ionization rate }
\end{aligned}
$$

Ignoring for a moment the collision ionization, the above equation can be numerically solved for a given set of initial condition. Assuming the temperature of the plasma $T=10^{5} \mathrm{~K}$, the initial density $n=10^{k}$ with $k=12$ and 13 and $\gamma=1.58 \times 10^{-2} / \mathrm{sec}$, we have the following degrees of ionization $x$ at the earth's distance for the initial degrees of ionization $x_{1}=0.6$, 0.8 and 0.9 .

\begin{tabular}{ccc}
$x_{1} / k$ & 12 & 13 \\
\hline 0.60 & 0.804 & 0.755 \\
0.80 & 0.896 & 0.825 \\
0.90 & 0.942 & 0.859
\end{tabular}

Clearly, the recombination process becomes more important for an initial number density of more than $n=10^{13} / \mathrm{cm}^{3}$, and such a value can be reasonably expected for the storm-producing plasma.

There have been a great number of studies on motions of prominence materials ejected from an active region of the sun. Since such observations are made by an $H_{a}$ filter, bright materials seen in solar photographs must be clouds of neutral hydrogen atoms ascending from the chromospheric region. Their motions have been traced in great detail to an altitude of $1 / 2$ solar radii. It is common to observe such bright materials for more than 30 min in the coronal region, so that clouds of neutral hydrogen atoms can definitely survive as a group for at least 30 min even in the intense radiation field at such an altitude, together with perhaps an intense bombardment by coronal electrons. Since the plasma causing geomagnetic storms should have a higher speed than some of those prominence materials, it is expected to escape from such a 'dangerous' region more rapidly.

It is interesting to note also that the magnitude of the main phase decrease shows a

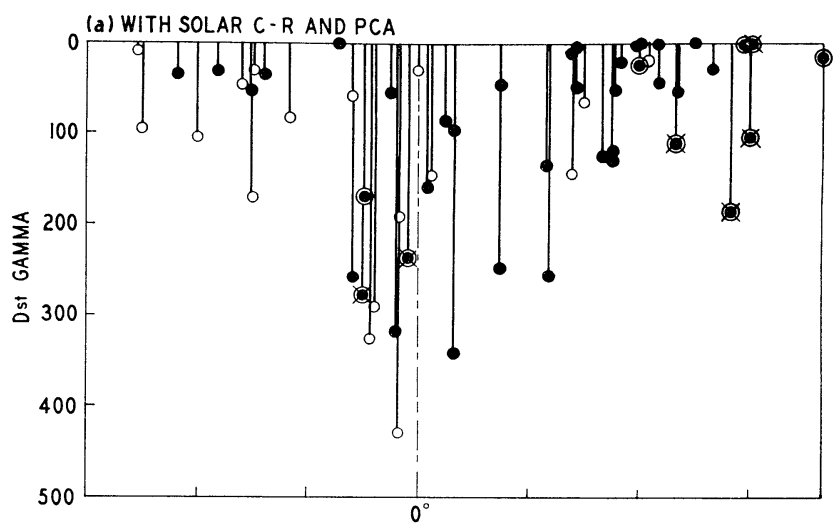

Fig. 9 The distribution of the magnitude of the main phase of magnetic storms associated with energetic solar particle events. 
remarkable center-limb effect, but no definite east-west asymmetry is found; Figure 9 (Yoshida and Akasofu (1965)). Therefore, it may be inferred that solar particles causing the main phase is not affected by the asymmetric configuration illustrated in Figure 8. This is another evidence to suggest that neutral hydrogen atoms are a possible source of energy for the ring current belt.

\section{Auroral and Polar Magnetic Substorms}

The flow pattern of the polar electrojet and of its return current in Figure 3 should be taken as showing only a gross average feature of the current system for the polar magnetic substorm. It appears intermittently a number of times during a single magnetic storm; the life time of the substorm is only of order a few hours. The current system in Figure 3 does not grow uniformly and simultaneously over the entire polar region, and the pattern itself changes greatly during a short life time of the substorm. Neither $S D$ nor $D S$ can express this intermittent nature of the substorms. The $D S$ variation with the storm time in the auroral zone can only be taken to be a sort of envelope of peak values of polar magnetic storm fields for an average storm. Further, Akasofu and Chapman (1963) have shown that the growth of the ring current and of the activity of polar magnetic substorms occur simultaneously. During a quiet initial phase, there is no particular enhancement of the substorms.

Such complexity of the substorm can only be well understood when the simultaneous and dynamical development of the auroral substorm is properly recognized. The auroral substorm is a violent dynamical process. It is most dramatically seen in the midnight sector as an explosive motion of the auroral system, such as an extremely rapid poleward motion of bright bands at an early stage in the midnight sector, westward traveling surges in the evening sector, and an eastward drift motion of the whole auroral system in the morning sector (Akasofu, 1964). During an intense magnetic substorm, the substorms occur quite frequently, so that a few well-defined substorms may be recognized during the course of a night; corresponding magnetic substorms (namely, negative bays) are also recognized.

In such a case, the whole auroral systems over the entire polar region rapidly repeats the following cycle

$$
\rightarrow \text { quiet phase } \rightarrow \text { active phase } \rightarrow \text { recovery phase- }
$$

and the corresponding auroral forms in the auroral zone in the midnight and morning sectors are

$$
\rightarrow \text { homogenous arc } \rightarrow \text { rayed bands } \rightarrow \text { patches- }
$$

Such a large-scale auroral activity over the entire polar region is interpreted as a manifestation of dynamical processes taking place in the magnetosphere. This situation is analogous to a display on the screen of a cathode ray tube, the polar atmosphere being the screen. In terms of such a projected image on the polar atmosphere, we seek to understand various aspects of both auroral and magnetic substorms and corresponding magnetospheric processes. Polar magnetic substorms are now understood to be simultaneous, but different 
manifestations of large-scale magnetospheric processes (Akasofu, 1965); some basic concepts in these problems have already been discussed by several workers including Piddington (1964), Hines (1964), Cole (1961), Fejer (1964) and others. Since the flow lines of the polar electrojet can be taken to be anti-parallel to flow lines of ionospheric electrons participating in a large-scale $(E \times B)$ motion of the magnetospheric plasma, the westward oval flow of the polar electrojet suggests a violent, but an intermittent eastward motion of a shell of the magnetospheric plasma near the boundary of the co-rotating region of the magnetosphere.

The cause of such a large-scale magnetospheric plasma motion is an important problem. The first visible indication of the auroral substorm appears in the midnight sector of the auroral zone as a sudden brightening of one of the quiet arcs; the substorm develops most extensively when the southernmost arc (in the northern hemisphere) is activated first. Further, all the dynamical features of the aurora associated with the substorm are propagated in all directions from the midnight sector; for example, westward traveling surges travel to as far as the early afternoon sector from the midnight sector. It is also obvious in Figure 3 that the polar electrojet is originally driven in the midnight sector. Thus Akasofu (1964) has suggested that the auroral substorm and the above eastward motion of the magnetospheric plasma shell are initiated in the midnight sector by an internal instability of the magnetospheric system, rather than by a direct interaction between the solar plasma with the magnetosphere; in fact, it is interesting to note that most intense substorms occur most frequently during the growing stage of the ring current belt (Akasofu and Chapman, 1963).

\section{Acknowledgement}

This research was supported in part by a grant from the National Aeronautics and Space Administration (NsG 201-62) and in part by a grant from the National Science Foundation (GP-2721).

\section{References}

Akasofu, S.-I., A source of the energy for geomagnetic storms and auroras, Planet. Space. Sci., 12, 801833, 1964.

Akasofu, S.-I., The development of the auroral substorm, Planet. Space Sci., 12, 272-282, 1964.

Akasofu, S.-I., The neutral hydrogen flux in the solar plasma flow-I Planet. Space Sci., 12, 905-913, 1964.

Akasofu, S.-I., Dynamic morphology of auroras, Space Sci. Rev., 4, 498-540, 1965.

Akasofu, S.-I. and Chapman, S., A neutral line discharge theory of the aurora polaris, Phil. Trans. Roy. Soc. London, A, 253, 359-406, 1961.

Akasofu, S.-I. and Chapman, S., The development of the main phase of great storms, J. Geophys. Res., 68, 125-129, 1963.

Akasofu, S.-I. and Chapman, S., Magnetic storms: the simultaneous development of the main phase $(D R)$ and of polar magnetic storms (DP). J. Geophys. Res., 68, 3155-3158, 1963.

Akasofu, S.-I. and Chapman, S., On the asymmetric development of magnetic storm fields in low and middle latitudes, Planet. Space Sci., 12, 607-626, 1964.

Akasofu, S.-I., Chapman, S. and Meng, C.-I., The polar electrojet, J. Atmosph. Terr. Phys., 27, 1275-1305, 1965.

Akasofu, S.-I., Chapman, S. and Venkatesan, D., The main phase of great magnetic storms, J. Geophys. 
Res., 68, 3345-3350.

Bell, B., Major flares and geomagnetic activity, Smithsonian Contrib. Astrophys. 5, 69-83, 1961.

Chapman, S., An outline of a theory of magnetic storms, Rroc. Roy. Soc., A, 95, 61-83, 1919.

Chapman, S., On certain average characteristics of world-wide magnetic disturbance, Proc. Roy. Soc. Lond., A., 115, 242-267, 1927.

Chapman, S., The electric current systems of magnetic storms, Terr. Magn. Atmos. Elect. 40, 349-370, 1935.

Chapman, S., The morphology of geomagnetic storms, An extension of the analysis of $D S$, the disturb ance local-time inequality. Annali de Geofisica, 5, 481-499, 1952.

Cole, K.D., On solar wind generation of polar geomagnetic disturbance, Geophy. J. RAS, 6, 103-114, 1961.

Cole, K.D., On the depletion of ionization in the outer magnetosphere during magnetic disturbances, $J$. Geophys. Res. 69, 3595-3601, 1964.

Dessler, A.J., Hanson, W.B. and Parker, F.N., Formation of the geomagnetic storm main phase ring current, J. Geophys. Res. 66, 3631-3637, 1961.

Fejer, J.A. Theory of the geomagnetic daily disturbance variations, J. Geophys. Res., 69, 123-137, 1964.

Haurwitz, M.W. Yoshida, S. and Akasofu, S.-I., Interplanetary magnetic field asymmetries and their effects on polar cap absorptions and Forbush decreases, J. Geophys. Res. 70, 2977-2988, 1965.

Hines, C.O., Hydromagnetic motions in the magnetosphere, Space Sci. Rev., 3, 342-379, 1964.

Newton, H.W. and Milson, A.S., The distribution of great and small geomagnetic storms in the sunspot cycle, J. Geophys. Res., 59, 203-214, 1954.

Parker, E.N., Sudden expansion of the corona following a large solar flares and the attendant magnetic field and cosmic-ray effects, Astrophys. J. 133, 1014-1033, 1961.

Piddington, J.H., Geomagnetic storms, auroras and associated effects, Space Sci. Rev. 3, 724-780, 1964.

Snyder, C.W., Neugebauer, M. and Rao, u.R., JRL Tech. Rep. No. 32-514. California Institute of Technology, Pasadena, California, 1963.

Sugiura, M. and Chapman, S., The average morphology of geomagnetic storms with sudden commencements, Abh. Akad. Wiss. Gottingen, Math, -Phys. Kl. Sonderheft, Nr. 4, 53 pp, 1960.

Vestine, E.H., The disturbance field of magnetic storms, Trans. Washington Meeting, IUGG, Bull. No. 11, 360-381, 1940.

Wilson, C.R., Ph.D. Thesis, University of Alaska, 1963.

Yoshida, S. and Akasofu, S.-I., A study of the propagation of solar particles in interplanetary space, Planet. Space Sci., 13, 435-448, 1965. 